TRANSACTIONS OF THE

AMERICAN MATHEMATICAL SOCIETY

Volume 354, Number 10, Pages 3955-3971

S 0002-9947(02)03024-6

Article electronically published on May 21, 2002

\title{
A THEORY OF CONCORDANCE FOR NON-SPHERICAL 3-KNOTS
}

\author{
VINCENT BLANLCEIL AND OSAMU SAEKI
}

\begin{abstract}
Consider a closed connected oriented 3-manifold embedded in the 5 -sphere, which is called a 3 -knot in this paper. For two such knots, we say that their Seifert forms are spin concordant, if they are algebraically concordant with respect to a diffeomorphism between the 3-manifolds which preserves their spin structures. Then we show that two simple fibered 3-knots are geometrically concordant if and only if they have spin concordant Seifert forms, provided that they have torsion free first homology groups. Some related results are also obtained.
\end{abstract}

\section{INTRODUCTION}

In this paper, a closed $(n-2)$-connected oriented $(2 n-1)$-dimensional manifold embedded in the $(2 n+1)$-sphere $S^{2 n+1}$ will be called a $(2 n-1)$-knot, or simply a knot. A (2n-1)-knot is spherical, if the embedded manifold is abstractly homeomorphic to $S^{2 n-1}$. Throughout the paper, we shall work in the smooth category. All the homology and cohomology groups are understood to be with integer coefficients unless otherwise specified.

In the 1960's Kervaire 9] and Levine [11 gave a classification of concordance classes of spherical $(2 n-1)$-knots with $n \geq 2$. They have proved that there is a bijective correspondence between the concordance classes of such spherical knots and the Witt-equivalence classes of certain Seifert forms.

More recently, for simple fibered $(2 n-1)$-knots with $n \geq 3$, which are not necessarily spherical, a complete characterization of concordance has been given by the first author and Michel in [1. This has been done by using Seifert forms as in the case of spherical knots: more precisely, two simple fibered $(2 n-1)$-knots are concordant if and only if their Seifert forms with respect to the $(n-1)$-connected

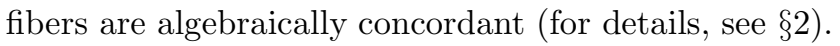

The case of 3-knots is much more difficult to study, since the dimension of the Seifert manifold is equal to four and the standard higher dimensional techniques, such as Whitney's trick, are not available. In [17], the second author has developed a theory of concordance for 3-knots which are homology 3 -spheres. In fact, such a

Received by the editors May 12, 2001 and, in revised form, February 15, 2002.

2000 Mathematics Subject Classification. Primary 57Q45; Secondary 57R40.

Key words and phrases. Concordance, 3-knot, Seifert form, algebraic concordance, spin structure, fibered knot.

The second author has been supported in part by Grant-in-Aid for Scientific Research (No. 11440022), Ministry of Education, Science and Culture, Japan, and was supported in part by Louis Pasteur University, France, during his stay there in September 2000.

(C)2002 American Mathematical Society 
case is very similar to the case of spherical 3-knots, which had been done by Levine [11].

In this paper, we shall try to develop a theory of concordance for 3-knots which are not necessarily homology 3 -spheres.

We shall first observe that each 3-knot carries a canonical spin structure induced from a Seifert manifold. For two 3-knots, we say that their Seifert forms are spin concordant, if they are algebraically concordant with respect to a diffemorphism which preserves their spin structures. Then we shall prove that two simple fibered 3 -knots with torsion free first homology groups are concordant if and only if they have spin concordant Seifert forms (see Theorem 2.15).

The paper is organized as follows. In $₫ 2$, we shall give more precise definitions to the notions introduced above, and state our main theorem.

In $\$ 3$, we shall show that spin concordance is a necessary condition for concordance. This will follow from the results already known in other dimensions together with a slight modification involving the spin structures. As corollaries, we give some interesting results which relate the concordance of 3-knots to their (image) regular homotopy classes, and to the isotopy classes of their punctured 3-knots.

In 44, we shall show that spin concordance is a sufficient condition for concordance. In fact, we shall prove this fact for simple 3-knots in general (not necessarily fibered). The main techniques used in the proof are mainly from 4-dimensional topology. In order to perform appropriate embedded surgeries as in the higher dimensional case, we shall have to find a set of embedded 2-spheres in a certain 4-manifold. The existence of such embedded spheres follows from Whitney's trick in higher dimensions, while in our dimension, the same argument fails. Thus we shall use a different method: we shall first construct embedded 2-spheres and then find a diffeomorphism which sends the embedded 2-spheres to others that represent correct homology classes.

In $₫ 5$, we shall extend the class of simple 3-knots for which our characterization of concordance continues to be valid. We also show that a similar extention is valid in higher dimensions as well.

In 6 , we shall restrict ourselves to embeddings of specific 3-manifolds. For example, we shall show that two embeddings of the connected sum of some copies of $S^{1} \times S^{2}$ into $S^{5}$ as simple fibered knots are concordant if and only if their Seifert forms are algebraically concordant; i.e., in this case, the spin strucutures are not necessary.

Finally in $\S 7$ we shall give some examples of simple 3-knots which have interesting properties.

The second author would like to thank the people at the Louis Pasteur University of Strasbourg, France, for their hospitality during his visit there.

\section{Preliminaries}

In this section, we shall give necessary definitions and then state our main theorem of this paper.

Definition 2.1. We say that two $(2 n-1)$-knots $K_{0}$ and $K_{1}$, abstractly diffeomorphic to the same $(2 n-1)$-dimensional manifold $\mathcal{K}$, are concordant, if there exists 
an embedding $\Phi: \mathcal{K} \times[0,1] \rightarrow S^{2 n+1} \times[0,1]$ such that

$$
\begin{aligned}
& \Phi(\mathcal{K} \times\{0\})=K_{0} \subset S^{2 n+1} \times\{0\}, \\
& \Phi(\mathcal{K} \times\{1\})=-K_{1} \subset S^{2 n+1} \times\{1\},
\end{aligned}
$$

where $-K_{1}$ is the mirror image of the knot $K_{1}$ with the orientation reversed (see Fig. 11).

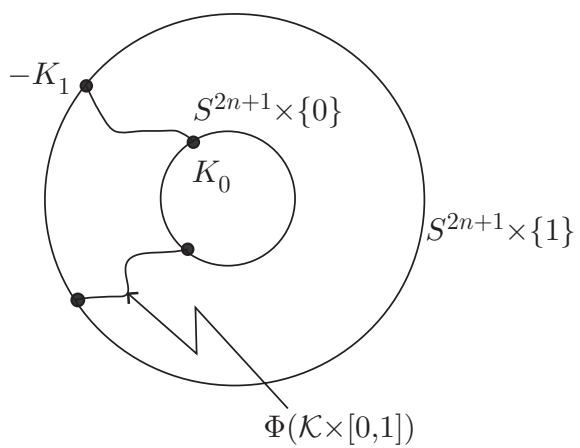

Figure 1. Concordance between $K_{0}$ and $K_{1}$

It is well-known that, for every $(2 n-1)$-knot $K$, there exists a compact oriented $2 n$-dimensional submanifold $F$ of $S^{2 n+1}$ having $K$ as the boundary. Such a manifold $F$ is called a Seifert manifold for $K$.

Definition 2.2. Suppose that $F$ is a compact oriented $2 n$-dimensional submanifold of $S^{2 n+1}$, and let $G$ be the quotient of $H_{n}(F)$ by its Z-torsion. The Seifert form associated with $F$ is the bilinear form $A: G \times G \rightarrow \mathbf{Z}$ defined as follows. For $(x, y) \in G \times G$, we define $A(x, y)$ to be the linking number in $S^{2 n+1}$ of $\xi$ and $\eta_{+}$, where $\xi$ and $\eta$ are $n$-cycles in $F$ representing $x$ and $y$ respectively, and $\eta_{+}$is the $n$-cycle $\eta$ pushed off $F$ into the positive normal direction to $F$ in $S^{2 n+1}$.

By definition a Seifert form for a $(2 n-1)$-knot $K$ is the Seifert form associated with a Seifert manifold for $K$.

Definition 2.3. A $(2 n-1)$-knot $K$ is fibered, if there exists a fibration $\phi: S^{2 n+1} \backslash$ $K \rightarrow S^{1}$ with $\phi$ being trivial on $U \backslash K$, where $U$ is a small open tubular neighborhood of $K$, such that the closure of each fiber is a Seifert manifold for $K$. We shall often call the closure of each fiber simply a fiber. The Seifert form defined on a fiber is always unimodular (for example, see [6]). In the following, for a fibered $(2 n-1)$ knot, we use the Seifert form defined on a fiber unless otherwise specified.

Definition 2.4. A $(2 n-1)$-knot is simple, if it admits an $(n-1)$-connected Seifert manifold. Furthermore, a fibered $(2 n-1)$-knot is simple, if its fiber is $(n-1)$ connected.

Recall that for higher-dimensional simple fibered knots we have a complete characterization of concordance using their Seifert forms as follows (see [1]).

Theorem 2.5. For $n \geq 3$, two simple fibered $(2 n-1)$-knots are concordant if and only if their Seifert forms with respect to the $(n-1)$-connected fibers are algebraically concordant. 
Here, algebraic concordance is defined as follows.

Definition 2.6. Let $\mathcal{A}$ be the set of all bilinear forms defined on free $\mathbf{Z}$-modules $G$ of finite rank. Set $\varepsilon=(-1)^{n}$. For $A \in \mathcal{A}$, let us denote by $A^{T}$ the transpose of $A$, by $S$ the $\varepsilon$-symmetric form $A+\varepsilon A^{T}$ associated with $A$, by $S^{*}: G \rightarrow G^{*}$ the adjoint of $S$ with $G^{*}$ being the dual $\operatorname{Hom}_{\mathbf{Z}}(G, \mathbf{Z})$ of $G$, and by $\bar{S}: \bar{G} \times \bar{G} \rightarrow \mathbf{Z}$ the $\varepsilon$-symmetric nondegenerate form induced by $S$ on $\bar{G}=G / \operatorname{Ker} S^{*}$. A submodule $M$ of $G$ is said to be pure, if $G / M$ is torsion free, or equivalently if $M$ is a direct summand of $G$. For a submodule $M$ of $G$, let us denote by $M^{\wedge}$ the smallest pure submodule of $G$ which contains $M$. We denote by $\bar{M}$ the image of $M$ in $\bar{G}$ by the natural projection map.

Definition 2.7. Let $A: G \times G \rightarrow \mathbf{Z}$ be a bilinear form in $\mathcal{A}$. The form $A$ is $W i t t$ associated to 0 , if the rank $m$ of $G$ is even and there exists a pure submodule $M$ of rank $m / 2$ in $G$ such that $A$ vanishes on $M$. Such a submodule $M$ is called a metabolizer for $A$.

Definition 2.8. Let $A_{i}: G_{i} \times G_{i} \rightarrow \mathbf{Z}, i=0,1$, be two bilinear forms in $\mathcal{A}$. Set $G=G_{0} \oplus G_{1}$ and $A=A_{0} \oplus-A_{1}$. The form $A_{0}$ is said to be algebraically concordant to $A_{1}$, if there exist a metabolizer $M$ for $A$ such that $\bar{M}$ is pure in $\bar{G}$, an isomorphism $\psi: \operatorname{Ker} S_{0}^{*} \rightarrow \operatorname{Ker} S_{1}^{*}$, and an isomorphism $\theta: \operatorname{Tors}\left(\operatorname{Coker} S_{0}^{*}\right) \rightarrow \operatorname{Tors}\left(\operatorname{Coker} S_{1}^{*}\right)$ which satisfy the following two conditions:

$$
\begin{aligned}
M \cap \operatorname{Ker} S^{*} & =\left\{(x, \psi(x)) ; x \in \operatorname{Ker} S_{0}^{*}\right\} \subset \operatorname{Ker} S_{0}^{*} \oplus \operatorname{Ker} S_{1}^{*}=\operatorname{Ker} S^{*}, \\
d\left(S^{*}(M)^{\wedge}\right) & =\left\{(y, \theta(y)) ; y \in \operatorname{Tors}\left(\operatorname{Coker} S_{0}^{*}\right)\right\} \\
& \subset \operatorname{Tors}\left(\operatorname{Coker} S_{0}^{*}\right) \oplus \operatorname{Tors}\left(\operatorname{Coker} S_{1}^{*}\right)=\operatorname{Tors}\left(\operatorname{Coker} S^{*}\right),
\end{aligned}
$$

where $d$ is the quotient map $G^{*} \rightarrow$ Coker $S^{*}$ and "Tors" means the torsion subgroup. In the above situation, we also say that $A_{0}$ and $A_{1}$ are algebraically concordant with respect to $\psi$ and $\theta$. Note that algebraic concordance is an equivalence relation, as has been shown in [1. Theorem 1].

Remark 2.9. In the above definition, if $A_{i}$ are Seifert forms associated with $(n-1)$ connected Seifert manifolds $F_{i}$ of simple $(2 n-1)$-knots $K_{i}, i=0,1$, then $\operatorname{Ker} S_{i}^{*}$ and Coker $S_{i}^{*}$ are naturally identified with $H_{n}\left(K_{i}\right)$ and $H_{n-1}\left(K_{i}\right)$ respectively, since we have the exact sequence

$$
\begin{aligned}
0=H_{n+1}\left(F_{i}, K_{i}\right) \rightarrow H_{n}\left(K_{i}\right) \rightarrow H_{n}\left(F_{i}\right) \stackrel{S_{i}^{*}}{\longrightarrow} H_{n}\left(F_{i}, K_{i}\right) & \rightarrow H_{n-1}\left(K_{i}\right) \rightarrow H_{n-1}\left(F_{i}\right)=0
\end{aligned}
$$

where we identify $H_{n}\left(F_{i}, K_{i}\right)$ with the dual of $H_{n}\left(F_{i}\right)$ by Poincaré-Lefschetz duality.

We know that algebraic concordance is necessary for concordance of simple fibered knots in any dimension (see [1]). In the case of 3-knots, we have to define a new equivalence relation for Seifert forms in order to characterize concordance classes of simple fibered 3-knots.

In the following, a spin structure on a manifold $X$ means the homotopy class of a trivialization of its tangent bundle over the 2-skeleton $X^{(2)}$. Note that $X$ admits a spin structure if and only if its second Stiefel-Whitney class $w_{2}(X)$ vanishes, and that if it admits one, then the set of all spin structures on $X$ is in one-to-one correspondence with $H^{1}\left(X ; \mathbf{Z}_{2}\right)$. 
Let $K$ be a 3-knot with Seifert manifold $F$. Recall that everything is oriented. Then $K$ has a natural normal 2-framing $\nu=\left(\nu_{1}, \nu_{1}\right)$ in $S^{5}$ such that the first normal vector field $\nu_{1}$ is obtained as the inward normal vector field of $K=\partial F$ in $F$. It is easy to show that the homotopy class of this 2-framing does not depend on the choice of the Seifert manifold $F$. Then $K$ carries a tangent 3 -framing on its 2-skeleton $K^{(2)}$ such that the juxtaposition with the above 2-framing gives the standard framing of $S^{5}$ restricted to $K^{(2)}$ up to homotopy. This means that $K$ carries a natural spin structure, which is determined uniquely up to homotopy. Furthermore, this spin structure coincides with that induced from the Seifert manifold $F$, which is endowed with the natural spin structure induced from $S^{5}$.

Definition 2.10. A 3-knot $K$ is said to be free, if $H_{1}(K)$ is torsion free over $\mathbf{Z}$.

Note that since Coker $S^{*}$ is torsion free for free knots, we do not need to consider condition (c2) in the definition of algebraic concordance (see Definition 2.8).

Definition 2.11. Consider two simple 3-knots $K_{0}$ and $K_{1}$. Let $A_{0}$ and $A_{1}$ be the Seifert forms of $K_{0}$ and $K_{1}$ respectively with respect to 1-connected Seifert manifolds. We say that $A_{0}$ and $A_{1}$ are spin concordant, if there exists an orientation preserving diffeomorphism $h: K_{0} \rightarrow K_{1}$ such that

(1) $h$ preserves their spin structures, and

(2) $A_{0}$ and $A_{1}$ are algebraically concordant with respect to $h_{*}: H_{2}\left(K_{0}\right) \rightarrow$ $H_{2}\left(K_{1}\right)$ and $h_{*} \mid$ Tors $H_{1}\left(K_{0}\right):$ Tors $H_{1}\left(K_{0}\right) \rightarrow$ Tors $H_{1}\left(K_{1}\right)$, where we identify $H_{2}\left(K_{i}\right)$ and $H_{1}\left(K_{i}\right)$ with $\operatorname{Ker} S_{i}^{*}$ and Coker $S_{i}^{*}$ respectively for $i=0,1$ (see Remark 2.9).

Note that if $K_{0}$ and $K_{1}$ are free 3 -knots, then we do not need to consider condition (c2) of Definition 2.8 and hence the isomorphism $h_{*} \mid$ Tors $H_{1}\left(K_{0}\right)$ in the above definition.

Remark 2.12. Spin concordance of Seifert forms with respect to 1-connected Seifert manifolds is an equivalence relation. More precisely, it defines an equivalence relation on the set of pairs of a Seifert manifold of a simple 3-knot and its Seifert form. This fact can be shown by using [1, Theorem 1] together with an additional argument concerning spin structures.

Remark 2.13. Algebraic concordance of Seifert forms does not necessarily imply spin concordance. See Examples 7.1 and 7.2 in $\$ 7]$

Remark 2.14. It is essential that we use 1-connected Seifert manifolds in Definition 2.11] See Example 7.5]

The main theorem of this paper is the following.

Theorem 2.15. Two simple fibered free 3-knots are concordant if and only if their Seifert forms with respect to 1-connected fibers are spin concordant.

We shall restrict ourselves to free 3-knots for two reasons. The first one is that algebraic concordance of Seifert forms is easier for free knots, since condition (c2) is not necessary in the definition of algebraic concordance. The second, and the most important one, is the dimension of the fiber. We shall use some results from 4-dimensional topology which require that the boundary 3-manifolds should have torsion free first homology groups. 


\section{SPIN CONCORDANCE AS A NECESSARY CONDITION FOR KNOT CONCORDANCE}

In this section, we shall prove the following, which is valid for simple fibered 3 -knots in general (not necessarily free).

Proposition 3.1. If two simple fibered 3-knots are concordant, then their Seifert forms with respect to 1-connected fibers are spin concordant.

Proof. Let $K_{0}$ and $K_{1}$ be two concordant simple fibered 3-knots. We denote by $F_{0}$ and $F_{1}$ their 1-connected fibers, and by $A_{0}$ and $A_{1}$ the associated Seifert forms, respectively. By [1], we know that $A_{0}$ and $A_{1}$ are algebraically concordant, and the metabolizer $M$ for $A_{0} \oplus-A_{1}$ is constructed as follows.

Let us denote by $\mathcal{S}$ the product $S^{5} \times[0,1]$. The definition of concordance (see Definition 2.1) gives a submanifold $C=\Phi(\mathcal{K} \times[0,1])$ of $\mathcal{S}$ such that $C \cap\left(S^{5} \times\{0\}\right)=$ $K_{0}$ and $C \cap\left(S^{5} \times\{1\}\right)=-K_{1}$. Set $N=F_{0} \cup C \cup\left(-F_{1}\right)$. By construction $N$ is a closed oriented 4-dimensional submanifold of $\mathcal{S}$. By classical obstruction theory, we see that there exists a compact oriented 5 -dimensional submanifold $W$ of $\mathcal{S}$ such that $N=\partial W$. Let $\lambda_{i}: H_{2}\left(F_{i}\right) \rightarrow H_{2}(N), i=0,1$, be the homomorphisms induced by the inclusions $F_{i} \hookrightarrow N$, and let $j: H_{2}(N) \rightarrow H_{2}(W)$ be the homomorphism induced by the inclusion $N \hookrightarrow W$. Then the submodule $M=\lambda^{-1}\left(\operatorname{Ker} j^{\wedge}\right)$ is a metabolizer for $A_{0} \oplus-A_{1}$, where $\operatorname{Ker} j^{\wedge}$ is the smallest pure submodule containing Ker $j$ and $\lambda=\lambda_{0}-\lambda_{1}: G=H_{2}\left(F_{0}\right) \oplus H_{2}\left(F_{1}\right) \rightarrow H_{2}(N)$. In [1], it has been shown that $\bar{M}$ is pure in $\bar{G}$.

Using a normal 2-framing of $C$ in $\mathcal{S}$ induced from the inward normal vector field along $N=\partial W$ in $W$, we see that the diffeomorphism $h$ between $K_{0}$ and $K_{1}$ induced by $C$ preserves their spin structures.

Moreover, in [1], it has been shown that $A_{0}$ and $A_{1}$ are algebraically concordant with respect to $h_{*}: H_{2}\left(K_{0}\right) \rightarrow H_{2}\left(K_{1}\right)$ and $h_{*} \mid$ Tors $H_{1}\left(K_{0}\right):$ Tors $H_{1}\left(K_{0}\right) \rightarrow$ Tors $H_{1}\left(K_{1}\right)$ by using the fact that $A_{0}$ and $A_{1}$ are unimodular.

Thus spin concordance is a necessary condition for concordance in the case of simple fibered 3-knots.

Remark 3.2. For a 3-knot $K$, a Seifert manifold is called an almost fiber of $K$, if its Seifert form is unimodular. A simple 3-knot is called a simple almost fibered 3-knot, if it admits a 1-connected almost fiber as a Seifert manifold (see [18, Remark 2.8 (1)], [4]). From the above proof, we easily see that Proposition 3.1 holds for simple almost fibered 3-knots in general.

As a corollary to Proposition 3.1 we have the following.

Corollary 3.3. Let $K$ be a simple almost fibered 3 -knot. Then the spin concordance class of a Seifert form with respect to a 1-connected almost fiber does not depend on the choice of the almost fiber of $K$.

Note that a 1-connected (almost) fiber is not necessarily unique for a simple 3 -knot (see [18, §5]).

As a corollary to the proof of Proposition 3.1, we have the following.

Corollary 3.4. If two 3-knots (which may not necessarily be fibered or almost fibered) are concordant, then they are (image) regularly homotopic as immersed manifolds in $S^{5}$.

Compare the above corollary with [13, Theorem 7]. 
Proof of Corollary [3.4 By [20], the regular homotopy class of an embedding of an oriented 3-manifold into $S^{5}$ is completely determined by two invariants: the $\mathrm{Wu}$ invariant and the signature of a Seifert manifold. If two 3-knots are concordant, then by using the argument in the proof of Proposition 3.1] we can show that their spin structures coincide with each other and that their Seifert manifolds have the same signature. Since the Wu invariant is determined by the spin structure, the two embeddings have the same $\mathrm{Wu}$ invariant. Hence they are (image) regularly homotopic as immersions into $S^{5}$.

Note that the converse of the above corollary does not hold, since there exist a lot of pairs of forms with the same signature but with distinct algebraic concordance class. For example, consider the simple spherical 3-knots with Seifert forms $A_{k}$, $k \geq 1$, defined in [11, p. 243]. Then they are all (image) regularly homotopic, but are pairwise nonconcordant.

Let $K$ be a 3 -knot. We shall denote by $K^{\circ}$ the punctured 3-manifold $K \backslash$ Int $D^{3}$ embedded in $S^{5}$. Then we have the following corollary to the proof of Proposition 3.1 as well.

Corollary 3.5. If two free 3 -knots $K_{0}$ and $K_{1}$ are concordant, then $K_{0}^{\circ}$ and $K_{1}^{\circ}$ are isotopic in $S^{5}$.

Proof. By using the manifold $C$ in the proof of Proposition 3.1 we can show that the Seifert forms of $K_{0}^{\circ}$ and $K_{1}^{\circ}$ with respect to their normal framings determined by their Seifert manifolds coincide with each other (see [19]). Then by [19], we see that the punctured 3-manifolds are isotopic.

\section{Spin CONCORDANCE AS A SUFFICIENT CONDItion FOR KNOT CONCORDANCE}

In this section, we shall prove the following, which is valid for simple free 3-knots in general, which may not be fibered.

Theorem 4.1. Consider two simple free 3-knots. If their Seifert forms with respect to 1-connected Seifert manifolds are spin concordant, then the 3-knots are concordant.

Proof. Let $K_{0}$ and $K_{1}$ be simple free 3-knots such that the Seifert forms $A_{0}$ and $A_{1}$ with respect to their 1-connected Seifert manifolds $F_{0}$ and $F_{1}$, respectively, are spin concordant. Let $M$ be the metabolizer and $h: K_{0} \rightarrow K_{1}$ the diffeomorphism as in Definitions 2.8 and 2.11 respectively. Set $F=F_{0} \natural\left(-F_{1}\right)$ and $V=\left(K_{0} \backslash\right.$ $\left.\operatorname{Int} D^{3}\right) \times[0,1]$, where the symbol "q" means a boundary connected sum. Note that $\partial F=K_{0} \sharp\left(-K_{1}\right)$ and $\partial V=K_{0} \sharp\left(-K_{0}\right)$, where the symbol "\#" means a usual connected sum (see Fig. 22and Fig. 3).

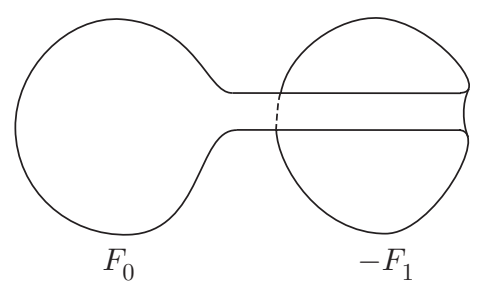

Figure 2. $F=F_{0} \natural\left(-F_{1}\right)$ 


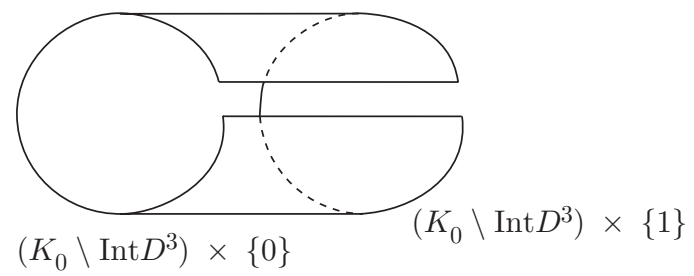

Figure 3. $V=\left(K_{0} \backslash \operatorname{Int} D^{3}\right) \times[0,1]$

Note also that the compact 4-manifold $V$ is spin, where the spin structure is induced from $K_{0}$. In the following, a spin surgery along a simple closed curve $c$ in a spin 4-manifold is a process of taking off the tubular neighborhood $N(c) \cong S^{1} \times D^{3}$ of $c$ and replacing it with $D^{2} \times S^{2}$ by gluing it along the boundary so that the resulting 4-manifold is spin and so that the spin structure on the exterior of $c$ coincides with the original one.

Lemma 4.2. For some integer $k \geq 0$, there exist a compact 4-manifold $\widetilde{V}$ and a diffeomorphism $\tilde{h}: F \sharp k\left(S^{2} \times S^{2}\right) \rightarrow \widetilde{V}$ such that

(1) $\widetilde{V}$ is obtained from $V$ by spin surgeries along simple closed curves, and

(2) $\tilde{h} \mid \partial\left(F \sharp k\left(S^{2} \times S^{2}\right)\right)=\operatorname{id}_{K_{0}} \sharp h^{-1}: K_{0} \sharp\left(-K_{1}\right) \rightarrow K_{0} \sharp\left(-K_{0}\right)$.

Proof. Step 1 . Since $H_{1}(V) \cong H_{1}\left(K_{0}\right)$ is a finitely generated free abelian group, we can obtain a 4-manifold $V_{1}$ with $H_{1}\left(V_{1}\right)=0$ from $V$ by spin surgeries along a finite set of simple closed curves $c_{i}, 1 \leq i \leq r=\operatorname{rank} H_{1}\left(K_{0}\right)$, representing a basis of $H_{1}(V)$.

Step 2. Since $\pi_{1}\left(V_{1}\right)$ is finitely generated, we can obtain a simply connected 4-manifold $V_{2}$ from $V_{1}$ by some spin surgeries.

Step 3. Since we have assumed that $H_{1}\left(K_{i}\right)$ is a free abelian group, the intersection forms of $F$ and $V_{2}$ are direct sums of a unimodular form and a zero form, where the dimensions of the null spaces are equal to the rank of $H_{1}\left(K_{0} \sharp\left(-K_{1}\right)\right) \cong$ $H_{1}\left(K_{0} \sharp\left(-K_{0}\right)\right)$. Furthermore, since they are spin, their intersection forms are of even type. Finally, since the Seifert forms of $F_{0}$ and $F_{1}$ are algebraically concordant, the signature of $F=F_{0} \downarrow\left(-F_{1}\right)$ vanishes, and that of $V_{2}$ is equal to that of $V$, which is zero. Thus, by the algebraic classification of unimodular forms (see, for example, [12]), by repeating some spin surgeries along trivial simple closed curves in $V_{2}$ if necessary, we may assume that there exists an isometry $\Lambda: H_{2}\left(F^{(k)}\right) \rightarrow H_{2}\left(V_{2}\right)$ for some integer $k \geq 0$ such that the diagram

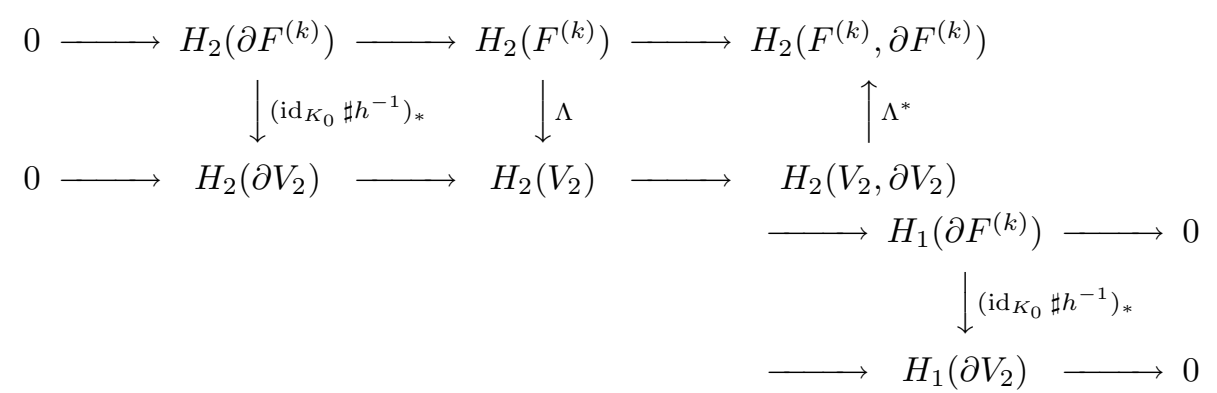




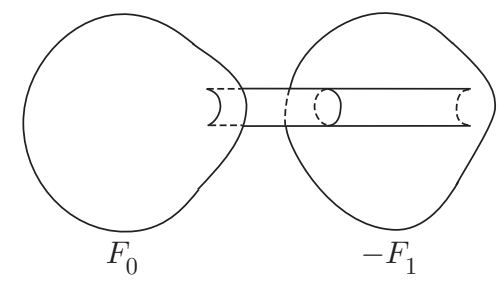

FiguRE $4 . F=F_{0} \sharp\left(-F_{1}\right)$

is commutative, where $F^{(k)}=F \sharp k\left(S^{2} \times S^{2}\right)$, we use Poincaré-Lefschetz duality to identify $H_{2}\left(F^{(k)}, \partial F^{(k)}\right)$ and $H_{2}\left(V_{2}, \partial V_{2}\right)$ with the duals of $H_{2}\left(F^{(k)}\right)$ and $H_{2}\left(V_{2}\right)$ respectively, and $\Lambda^{*}$ is the adjoint of $\Lambda$.

Step 4 . Note that the spin structures of $K_{0} \sharp\left(-K_{1}\right)$ and $K_{0} \sharp\left(-K_{0}\right)$ coincide with those induced from $F^{(k)}$ and $V_{2}$ respectively. Thus $F^{(k)} \cup_{\mathrm{id}_{K_{0}} \sharp h^{-1}}\left(-V_{2}\right)$ is a closed spin 4-manifold, since $\operatorname{id}_{K_{0}} \sharp h^{-1}$ preserves the spin structures by our hypothesis. Furthermore, $K_{0} \sharp\left(-K_{1}\right)$ and $K_{0} \sharp\left(-K_{0}\right)$ are connected. Then by an argument of Boyer [2 p. 347], we see that there exists a smooth $h$-cobordism relative to the boundary between $F^{(k)}$ and $V_{2}$ such that the induced diffeomorphism between the boundaries of $F^{(k)}$ and $V_{2}$ coincides with $\operatorname{id}_{K_{0}} \sharp h^{-1}$, and such that the induced isomorphism between $H_{2}\left(F^{(k)}\right)$ and $H_{2}\left(V_{2}\right)$ coincides with $\Lambda$ above.

Step 5 . Finally, by the 5 -dimensional stable $h$-cobordism theorem due to Lawson [10] and Quinn [14, we see that there exists a diffeomorphism between $F^{\left(k+k^{\prime}\right)}=$ $F^{(k)} \sharp k^{\prime}\left(S^{2} \times S^{2}\right)$ and $\widetilde{V}=V_{2} \sharp k^{\prime}\left(S^{2} \times S^{2}\right)$ extending $\operatorname{id}_{K_{0}} \sharp h^{-1}: \partial F^{(k)} \rightarrow \partial V_{2}$. Since $\widetilde{V}$ can be obtained from $V_{2}$ by repeating $k^{\prime}$ times the spin surgeries along trivial simple closed curves, we get the result. This completes the proof of Lemma 4.2

Remark 4.3. In Step 1, we can choose the curves $c_{i}, 1 \leq i \leq r$, inside $\left(K_{0} \backslash \operatorname{Int} D^{3}\right) \times$ $\{1 / 2\}$. After the surgeries, the embedded 2 -sphere $\Sigma_{i}$ in $V_{1}$ corresponding to the center sphere $\{0\} \times S^{2}$ of the piece $D^{2} \times S^{2}$ replacing $N\left(c_{i}\right)$ is homologous to the boundary of a meridian 3-disk of $c_{i}$ in $V$. Let $\gamma_{i}^{*}, 1 \leq i \leq r$, be a basis of $H_{2}\left(K_{0} \backslash \operatorname{Int} D^{3}\right) \cong H_{2}\left(K_{0}\right)$ which is Poincaré dual to the basis $\left[c_{i}\right], 1 \leq i \leq r$, of $H_{1}\left(K_{0}\right)$, where $[*]$ denotes the homology class represented by $*$. Then, by the above observation, we have $\left[\Sigma_{i}\right]=i_{0 *} \gamma_{i}^{*}-i_{1 *} \gamma_{i}^{*}$, where $i_{0}: K_{0} \rightarrow K_{0} \times\{0\} \subset V_{1}$ and $i_{1}: K_{0} \rightarrow K_{0} \times\{1\} \subset V_{1}$ denote the inclusions.

Lemma 4.4. For some integer $k \geq 0$, there exist a compact 4-manifold $\widetilde{V^{\prime}}$ and a diffeomorphism $\tilde{h^{\prime}}: F_{0} \sharp\left(-F_{1}\right) \sharp k\left(S^{2} \times S^{2}\right) \rightarrow \widetilde{V^{\prime}}$ such that

(1) $\widetilde{V^{\prime}}$ is obtained from $V^{\prime}=K_{0} \times[0,1]$ by spin surgeries along simple closed curves, and

(2) $\tilde{h^{\prime}} \mid \partial\left(F_{0} \sharp\left(-F_{1}\right) \sharp k\left(S^{2} \times S^{2}\right)\right)=\operatorname{id}_{K_{0}} \amalg h^{-1}: K_{0} \amalg\left(-K_{1}\right) \rightarrow K_{0} \amalg\left(-K_{0}\right)$.

Proof. Just glue $D^{3} \times[0,1]$ to $F \sharp k\left(S^{2} \times S^{2}\right)$ and $\widetilde{V}$ in Lemma 4.2 along $\partial D^{3} \times[0,1]$ to obtain $F_{0} \sharp\left(-F_{1}\right) \sharp k\left(S^{2} \times S^{2}\right)$ and $\widetilde{V^{\prime}}$ respectively (see Fig. 2 and Fig. 4).

Let $\widetilde{V^{\prime}}$ be as in the above lemma, i.e., obtained from $K_{0} \times[0,1]$ by some spin surgeries. Let $\Sigma_{1}, \Sigma_{2}, \ldots, \Sigma_{r}$ be the embedded 2 -spheres in $\widetilde{V^{\prime}}$ which have been created in the course of the surgeries in Step 1 of the proof of Lemma 4.2 (for details, see Remark 4.3). Furthermore, let $\Sigma_{r+1}, \Sigma_{r+2}, \ldots, \Sigma_{r+s}$ be the 2-spheres 
in $\widetilde{V^{\prime}}$ created in Steps 2-5 in the proof of Lemma 4.2. For the latter spheres, since the surgery curves are all null homologous, we see that there exist homology classes $\sigma_{r+1}^{*}, \sigma_{r+2}^{*}, \ldots, \sigma_{r+s}^{*} \in H_{2}\left(\widetilde{V^{\prime}}\right)$ such that

$$
\left[\Sigma_{i}\right] \cdot \sigma_{j}^{*}= \begin{cases}1, & i=j \\ 0, & i \neq j\end{cases}
$$

for $r+1 \leq i, j \leq r+s$. Modifying $\sigma_{i}^{*}, r+1 \leq i \leq r+s$, appropriately, we may further assume that the $s$ submodules $\left\langle\left[\Sigma_{i}\right], \sigma_{i}^{*}\right\rangle$ are orthogonal to each other with respect to the intersection form $\widetilde{S}$ of $\widetilde{V^{\prime}}$, and that the intersection matrix of $\left\langle\left[\Sigma_{i}\right], \sigma_{i}^{*}\right\rangle$ is equal to

$$
\left(\begin{array}{ll}
0 & 1 \\
1 & 0
\end{array}\right)
$$

where for a subset $X$ of a module, $\langle X\rangle$ denotes the submodule generated by $X$. Note also that

$$
H_{2}\left(\widetilde{V^{\prime}}\right)=\operatorname{Ker} \widetilde{S}^{*} \oplus\left(\bigoplus_{i=r+1}^{r+s}\left\langle\left[\Sigma_{i}\right], \sigma_{i}^{*}\right\rangle\right),
$$

where the symbol " $\bigoplus^{\perp}$ " denotes an orthogonal direct sum.

By taking the connected sum of $k$ copies of $S^{2} \times S^{2}$ with $F_{0}$ inside $S^{5}$, we may assume that $A_{0}$ is the Seifert form with respect to $F_{0} \sharp k\left(S^{2} \times S^{2}\right)$. Let $A_{1}$ be the Seifert form with respect to $F_{1}$. Furthermore, let $S$ be the symmetric form associated with $A_{0} \oplus\left(-A_{1}\right)$ (see Definition [2.6). Note that $S$ can be naturally identified with the intersection form of $F_{0} \sharp\left(-F_{1}\right) \sharp k\left(S^{2} \times S^{2}\right)$ and hence with that of $\widetilde{V^{\prime}}$. In the following, we shall identify $F_{0} \sharp\left(-F_{1}\right) \sharp k\left(S^{2} \times S^{2}\right)$ with $\widetilde{V^{\prime}}$ by using $\tilde{h^{\prime}}$ in Lemma 4.4

Lemma 4.5. There exists an isometry $\Phi$ of $H_{2}\left(F_{0} \sharp\left(-F_{1}\right) \sharp k\left(S^{2} \times S^{2}\right)\right)$ with respect to $S$ such that

(1) $\Phi \mid \operatorname{Ker} S^{*}=\mathrm{id}$, and

(2) $\Phi_{*}\left[\Sigma_{1}\right], \Phi_{*}\left[\Sigma_{2}\right], \ldots, \Phi_{*}\left[\Sigma_{r+s}\right]$ are generators of the metabolizer $M$.

Proof. First recall that $\left[\Sigma_{1}\right],\left[\Sigma_{2}\right], \ldots,\left[\Sigma_{r}\right]$ lie in Ker $S^{*}$ by Remark 4.3

As has been shown in [1, Proposition 2.1], there exist a basis $\left\{m_{i}, m_{i}^{*} ; i=\right.$ $1,2, \ldots, r+s\}$ of $G=H_{2}\left(F_{0} \sharp\left(-F_{1}\right) \sharp k\left(S^{2} \times S^{2}\right)\right)$ such that

(a) $\left\{m_{i} ; i=1,2, \ldots, r+s\right\}$ is a basis of $M$,

(b) $\left\{m_{i}, m_{i}^{*} ; i=1,2, \ldots, r\right\}$ is a basis of $\operatorname{Ker} S^{*}$ and $\left\{m_{i}^{*} ; i=1,2, \ldots, r\right\}$ is a basis of $\operatorname{Ker} S_{0}^{*}$, where $S_{0}$ is the symmetric form associated with $A_{0}$, and

(c) the submodules $\left\langle m_{i}, m_{i}^{*}\right\rangle, i=1,2, \ldots, r+s$, are orthogonal for $S$; i.e.,

$$
G=\bigoplus_{i=1}^{r+s}\left\langle m_{i}, m_{i}^{*}\right\rangle
$$

We may further assume that

$$
S\left(m_{i}, m_{i}\right)=0, \quad S\left(m_{i}, m_{i}^{*}\right)=1, \quad S\left(m_{i}^{*}, m_{i}^{*}\right)=0
$$

for $r+1 \leq i \leq r+s$, since Coker $S^{*}$ is torsion free. Then define the isometry $\Phi: G \rightarrow$ $G$ by $\Phi \mid \operatorname{Ker} S^{*}=\mathrm{id}, \Phi\left(\left[\Sigma_{i}\right]\right)=m_{i}$ and $\Phi\left(\sigma_{i}^{*}\right)=m_{i}^{*}$ for $i=r+1, r+2, \ldots, r+s$. This completes the proof of Lemma 4.5. 
Lemma 4.6. For some integer $k \geq 0$, there exists an orientation preserving selfdiffeomorphism $\varphi$ of the 4-manifold $F_{0} \sharp\left(-F_{1}\right) \sharp k\left(S^{2} \times S^{2}\right)$, which is the identity on the boundary, such that $\varphi_{*}=\Phi$ on the second homology group.

Proof. Let $J$ be the submodule of $G=H_{2}\left(F_{0} \sharp\left(-F_{1}\right) \sharp k\left(S^{2} \times S^{2}\right)\right)$ generated by $\left[\Sigma_{i}\right]$ and $\sigma_{i}^{*}$ with $r+1 \leq i \leq r+s$. Note that $G=\operatorname{Ker} S^{*} \oplus J$ and that the intersection matrix with respect to this decomposition is of the form $0 \oplus Q$, where $Q$ is a unimodular symmetric matrix of even type and zero signature.

Then it is not difficult to see that an arbitrary isometry of $\left(\operatorname{Ker} S^{*} \oplus J ; 0 \oplus Q\right)$ which is the identity on $\operatorname{Ker} S^{*}$ is a composition of the following isometries:

(a) $\operatorname{id} \oplus \Lambda$, where $\Lambda$ is an isometry of $(J ; Q)$, and

(b) an isometry represented by the matrix of the form

$$
\left(\begin{array}{cc}
\text { id } & * \\
0 & \text { id }
\end{array}\right)
$$

with respect to the decomposition $\operatorname{Ker} S^{*} \oplus J$.

We can easily realize isometries of type (a) by diffeomorphisms which are the identity on the boundary, by using Wall's argument [21], since we may assume $k \geq 1$.

In order to realize isometries of type (b), we need the following lemma.

Lemma 4.7. Increasing $k$ if necessary, we may assume that

$$
F_{0} \sharp\left(-F_{1}\right) \sharp k\left(S^{2} \times S^{2}\right) \backslash \Sigma_{i}
$$

is simply connected for $1 \leq i \leq r+s$.

Proof. Since $F_{0} \sharp\left(-F_{1}\right) \sharp k\left(S^{2} \times S^{2}\right)$ is simply connected, $\pi_{1}\left(F_{0} \sharp\left(-F_{1}\right) \sharp k\left(S^{2} \times S^{2}\right) \backslash \Sigma_{i}\right)$ is normally generated by a meridian $\mu_{i}$ of $\Sigma_{i}$, where $\mu_{i}$ is the boundary of a fiber of the 2-disk bundle neighborhood of $\Sigma_{i}$. Then, performing spin surgeries along $\mu_{i}$, we get the desired situation.

Now let us go back to the proof of Lemma 4.6 By Lemma 4.7, the spin surgery creating each $\Sigma_{i}$ corresponds to the connected sum operation with $S^{2} \times S^{2}$. Thus by [21, Theorem 1], we get a diffeomorphism realizing an isometry of type (b) corresponding to a matrix of the form

$$
\left(\begin{array}{cc}
\text { id } & E \\
0 & \text { id }
\end{array}\right)
$$

where $E$ is a matrix having one entry equal to 1 and all the others equal to zero. Using this type of diffeomorphisms (sometimes we have to interchange the two factors of $S^{2} \times S^{2}$, or use the inverse diffeomorphism), we get the desired diffeomorphism. This completes the proof of Lemma 4.6.

Thus we have proved that the embedded 2 -spheres $\varphi\left(\Sigma_{1}\right), \varphi\left(\Sigma_{2}\right), \ldots, \varphi\left(\Sigma_{r+s}\right)$ in $F_{0} \sharp\left(-F_{1}\right) \sharp k\left(S^{2} \times S^{2}\right)$ constitute a set of generators for the metabolizer $M$.

Recall that $F_{0} \sharp\left(-F_{1}\right) \sharp k\left(S^{2} \times S^{2}\right)$ is embedded in $\mathcal{S}=S^{5} \times[0,1]$. Then we can perform appropriate surgeries along these embedded 2 -spheres inside $\mathcal{S}$ as in [1, §4]. Since each surgery process is exactly the inverse operation of each spin surgery performed in the construction of $\widetilde{V^{\prime}}$ (modified by the diffeomorphism $\varphi$ ), the resulting 4-manifold is diffeomorphic to $K_{0} \times[0,1]$, which is embedded in $\mathcal{S}$. Thus $K_{0}$ and $K_{1}$ are concordant. This completes the proof of Theorem 4.1 and hence Theorem 2.15. 
Remark 4.8. As has been noted in Remark 2.13 algebraic concordance does not necessarily imply spin concordance. Hence, Theorem 4.1 does not hold if we replace spin concordance by algebraic concordance, even if we add the assumption that the 3-knots are abstractly diffeomorphic.

\section{Extension to a LARGER ClASS OF 3-KNOTS}

As the arguments of $\$ 4$ show, the sufficiency of Theorem 2.15 holds for simple free 3-knots: i.e., the 3-knots in question need not be fibered. However, for the proof of necessity in $\$ 2$ we have used the hypothesis that the 3-knots are fibered. In this section, we shall try to extend the class of simple fibered free 3-knots in such a way that the necessity continues to hold for a larger class of 3-knots.

First we give a definition which is valid for any dimension.

Definition 5.1. We say that a simple $(2 n-1)$-knot $K$ is $C$-algebraically fibered, if the Seifert form of $K$ with respect to an $(n-1)$-connected Seifert manifold is algebraically concordant to a unimodular form, where the zero form is also considered to be unimodular. In the following, for a $C$-algebraically fibered $(2 n-1)$-knot, we use the Seifert form defined on an $(n-1)$-connected Seifert manifold which is algebraically concordant to a unimodular form, unless otherwise specified. Note that simple fibered knots are always $C$-algebraically fibered.

Remark 5.2. A simple $(2 n-1)$-knot is said to be algebraically fibered, if the Seifert form with respect to an $(n-1)$-connected Seifert manifold is $S$-equivalent to a unimodular matrix (see [7], [16, §4]). Then we see easily that for a simple $(2 n-1)$ knot, we have

$$
\text { simple fibered } \Longrightarrow \text { algebraically fibered } \Longrightarrow C \text {-algebraically fibered. }
$$

Note that the reverse implications do not hold in general. See [7] and Example 7.3

Now, let us consider the case of 3 -knots. Recall that a stabilizer $K_{S}$ is a simple fibered spherical 3-knot whose fiber is diffeomorphic to $\left(S^{2} \times S^{2}\right) \sharp\left(S^{2} \times S^{2}\right) \backslash \operatorname{Int} D^{4}$. Such a stabilizer does exist. For details, see [16] §4]. Furthermore, there also exists a stabilizer which is null cobordant (see [15, p. 600] or [18 §10]). In the following, $K_{S}$ will denote such a null cobordant stabilizer.

Proposition 5.3. Let $K$ be a simple free 3-knot. If $K$ is $C$-algebraically fibered, then there exists a simple fibered free 3 -knot $K^{\prime}$ such that

(1) $K$ and $K^{\prime}$ are concordant, and

(2) the Seifert form of $K$ with respect to a 1-connected Seifert manifold and that of $K^{\prime}$ with respect to a 1-connected fiber are spin concordant.

Compare the above proposition with [16 Proposition 4.4].

Proof of Proposition 5.3 Let $F$ be a 1-connected Seifert manifold of $K$ and $A$ the Seifert form for $F$. Note that $A$ is algebraically concordant to a unimodular form $L$ by our assumption. Let $\psi: H_{2}(K)=\operatorname{Ker}\left(A+A^{T}\right) \rightarrow \operatorname{Ker}\left(L+L^{T}\right)$ be the isomorphism with respect to which $A$ and $L$ are algebraically concordant.

Let us first show that there exists a compact 1-connected oriented spin 4-manifold $F^{\prime}$ with boundary diffeomorphic to $K$ such that the spin structures induced from 
$F$ and $F^{\prime}$ on $K$ coincide with each other, and such that the intersection form of $F^{\prime}$ is isomorphic to

$$
\left(L+L^{T}\right) \oplus 2 k\left(\begin{array}{cc}
0 & 1 \\
1 & 0
\end{array}\right)
$$

for some $k \geq 0$. We can construct such a 4 -manifold $F^{\prime}$ as follows.

We first construct a 4-dimensional special handlebody $F_{1}$ consisting of one 0 handle and some 2-handles attached to the 0 -handle simultaneously such that $\partial F_{1}$ is diffeomorphic to $K, F_{1}$ is spin, and the spin structure induced from $F_{1}$ coincides with the given spin structure on $K$ (for details, see [5]). Then by Rohlin's theorem together with Novikov additivity for signature, the difference of the signatures of $F$ and $F_{1}$ must be divisible by 16 . Hence, by using some copies of a spin 4-dimensional special handlebody with boundary $S^{3}$ and with signature \pm 16 (see [5]), we may assume that $F$ and $F_{1}$ have the same signature. Note that the signature of $F$ is equal to that of $L+L^{T}$. Then by the classification of symmetric unimodular forms, we see that the intersection form of $F^{\prime}=F_{1} \sharp k^{\prime}\left(S^{2} \times S^{2}\right)$ is isomorphic to the form (5.1) for some $k, k^{\prime} \geq 0$. Here, we need the assumption that $H_{1}(K)$ is free.

Note that the above isomorphism between the intersection form of $F^{\prime}$ and the form (5.1) induces an isomorphism $H_{2}(K)=\operatorname{Ker}\left(A+A^{T}\right) \rightarrow \operatorname{Ker}\left(L+L^{T}\right)$. Changing the isomorphism between the intersection form of $F^{\prime}$ and (5.1) if necessary, we may assume that the induced isomorphism coincides with $\psi$.

Recall that $F^{\prime}$ has a handlebody decomposition consisting of one 0-handle and some 2-handles. Thus, by using Kervaire's argument [8, pp. 255-257], we can embed $F^{\prime}$ into $S^{5}$ so that its Seifert form is given by $L \oplus k L_{S}$, where $L_{S}$ is the Seifert form of a null cobordant stabilizer $K_{S}$ with respect to the 1-connected fiber. Set $K^{\prime}=\partial F^{\prime}$.

Since $L$ is unimodular, by using the stabilization technique developed in [16, §4], we may assume that $K^{\prime}$ is a simple fibered 3 -knot, increasing $k$ if necessary.

Note that the Seifert form $L \oplus k L_{S}$ for $K^{\prime}$ is algebraically concordant to $L$, which is algebraically concordant to the Seifert form $A$ for $K$ by our assumption. Furthermore, by the above construction, we see easily that $L \oplus k L_{S}$ and $A$ are spin concordant. Thus we have proved item (2) in the proposition. Item (1) then follows from Theorem 4.1. This completes the proof of Proposition 5.3 .

Corollary 5.4. If two C-algebraically fibered simple free 3-knots are concordant, then their Seifert forms with respect to 1-connected Seifert manifolds are spin concordant.

Proof. Let $K_{0}$ and $K_{1}$ be the simple free 3 -knots as above. Then by Proposition 5.3 $K_{0}$ and $K_{1}$ are concordant to simple fibered free 3 -knots $K_{0}^{\prime}$ and $K_{1}^{\prime}$ with spin concordant Seifert forms respectively. Then, since $K_{0}^{\prime}$ and $K_{1}^{\prime}$ are concordant, they have spin concordant Seifert forms by Proposition 3.1 Thus $K_{0}$ and $K_{1}$ have spin concordant Seifert forms, since spin concordance is an equivalence relation (see Remark 2.12). This completes the proof.

Combining the above corollary with Theorem 4.1 we get the following.

Theorem 5.5. Two $C$-algebraically fibered simple free 3 -knots are concordant if and only if their Seifert forms with respect to 1-connected Seifert manifolds are spin concordant.

Note that there are a lot of $C$-algebraically fibered simple free 3 -knots which are not fibered (see Example 7.3 of 97 ). 
We can prove a similar theorem for higher dimensions as well, as follows.

Theorem 5.6. For $n \geq 3$, two $C$-algebraically fibered simple $(2 n-1)$-knots are concordant if and only if their Seifert forms with respect to $(n-1)$-connected Seifert manifolds are algebraically concordant.

Proof. Replacing Proposition 3.1 in the argument for the 3-dimensional case by [1. Theorem $2^{\prime}$, we see that we have only to show the following: if a simple $(2 n-1)$ knot $K$ with $n \geq 3$ is $C$-algebraically fibered, then $K$ is concordant to a simple fibered $(2 n-1)$-knot $K^{\prime}$ such that the Seifert form $A$ of an $(n-1)$-connected Seifert manifold for $K$ is algebraically concordant to the Seifert form of an $(n-1)$-connected fiber of $K^{\prime}$.

Since $K$ is $C$-algebraically fibered, $A$ is algebraically concordant to a unimodular form $L$. By Durfee [3], such a form is realized as the Seifert form of a simple fibered $(2 n-1)$-knot $K^{\prime}$. Then by [1, Theorem 3], $K$ is concordant to $K^{\prime}$. This completes the proof.

\section{Special Cases}

So far, we had to consider spin concordance of Seifert forms instead of the usual algebraic concordance for 3 -knots. In this section, we shall show that in some special cases, algebraic concordance is sufficient.

Let us begin with the following definition.

Definition 6.1. Let $\mathcal{M}$ be a closed connected oriented 3-manifold. A 3-knot $K$ is called an $\mathcal{M}$-knot, if $K$ is abstractly diffeomorphic to $\mathcal{M}$, orientation preservingly.

For a closed connected oriented 3 -manifold $\mathcal{M}$, let us consider the following conditions.

(6.1) For any isomorphism $\psi: H_{2}(\mathcal{M}) \rightarrow H_{2}(\mathcal{M})$, there exists an orientation preserving diffeomorphism $h_{1}: \mathcal{M} \rightarrow \mathcal{M}$ such that $h_{1 *}=\psi$.

(6.2) For any two spin structures of $\mathcal{M}$, there exists an orientation preserving diffeomorphism $h_{2}: \mathcal{M} \rightarrow \mathcal{M}$ which sends one spin structure to the other and is such that $h_{2 *}: H_{2}(\mathcal{M}) \rightarrow H_{2}(\mathcal{M})$ is the identity.

Then we have the following.

Proposition 6.2. Let $\mathcal{M}$ be a closed connected oriented 3-manifold with torsion free first homology group. Suppose that the above conditions (6.1) and (6.2) are satisfied for $\mathcal{M}$. Then two $C$-algebraically fibered simple $\mathcal{M}$-knots are concordant if and only if their Seifert forms with respect to 1-connected Seifert manifolds are algebraically concordant.

Proof. The necessity follows from Corollary 5.4 .

Now, suppose that $K_{0}$ and $K_{1}$ are $C$-algebraically fibered simple $\mathcal{M}$-knots whose Seifert forms $A_{0}$ and $A_{1}$ with respect to 1-connected Seifert manifolds $F_{0}$ and $F_{1}$, respectively, are algebraically concordant. We suppose that $A_{0}$ and $A_{1}$ are algebraically concordant with respect to the isomorphism $\psi: H_{2}\left(K_{0}\right) \rightarrow H_{2}\left(K_{1}\right)$. By the conditions (6.1) and (6.2), we see that there exists an orientation preserving diffeomorphism $h: K_{0} \rightarrow K_{1}$ such that $h_{*}=\psi$ and $h$ sends the spin structure of $K_{0}$ to that of $K_{1}$. Hence, $A_{0}$ and $A_{1}$ are spin concordant with respect to $h$. Thus by Theorem 4.1, $K_{0}$ and $K_{1}$ are concordant. This completes the proof. 
For example, if $\mathcal{M}$ is a $\mathbf{Z}$-homology 3-sphere, i.e., if $H_{1}(\mathcal{M})=0$, then $H_{2}(\mathcal{M})=$ 0 and $\mathcal{M}$ admits a unique spin structure. Thus the conditions (6.1) and (6.2) are automatically satisfied. As another example, consider $\mathcal{M}=\sharp^{k}\left(S^{1} \times S^{2}\right)$, the connected sum of $k$ copies of $S^{1} \times S^{2}$ with $k \geq 1$. Then it is well known that the conditions (6.1) and (6.2) are satisfied also in this case. Thus we have the following.

Corollary 6.3. Suppose $\mathcal{M}$ is a Z-homology 3-sphere, or $\mathcal{M}=\sharp^{k}\left(S^{1} \times S^{2}\right), k \geq$ 1. Then two $C$-algebraically fibered simple $\mathcal{M}$-knots are concordant if and only if their Seifert forms with respect to 1-connected Seifert manifolds are algebraically concordant.

In fact, when $\mathcal{M}$ is a $\mathbf{Z}$-homology 3 -sphere, a stronger result is known. For details, see [17].

\section{EXAMPLES}

In this section, we give various examples with interesting properties.

Example 7.1. Set $\mathcal{M}=S^{1} \times \Sigma_{g}$, where $\Sigma_{g}$ is the closed connected orientable surface of genus $g \geq 2$. Note that $H_{1}(\mathcal{M})$ is torsion free. Let $K_{0}$ and $K_{1}$ be the simple fibered $\mathcal{M}$-knots constructed in [18, Proposition 3.8]. They have the property that their Seifert forms are isomorphic, but that there exists no diffeomorphism between $K_{0}$ and $K_{1}$ which preserves their spin structures. Thus, the Seifert forms of $K_{0}$ and $K_{1}$ are algebraically concordant, but are not spin concordant. Hence they are not concordant by Proposition 3.1

This example shows that the spin structure plays an essential role in the theory of concordance for 3-knots.

Example 7.2. Let $\mathcal{M}$ be a nontrivial orientable $S^{1}$-bundle over the closed connected orientable surface of genus $g \geq 2$. Note that $H_{1}(\mathcal{M})$ is not torsion free in general. Let $K_{1}, K_{2}, \ldots, K_{n}$ be the simple fibered $\mathcal{M}$-knots constructed in [18] Theorem 3.1]. They have the property that their Seifert forms are isomorphic to each other, but that any such isomorphism restricted to $H_{2}\left(K_{i}\right)$ cannot be realized by a diffeomorphism. Thus, the Seifert forms of $K_{i}$ are algebraically concordant to each other, but are not spin concordant. Hence they are not concordant by Proposition 3.1, which is valid also for non-free simple fibered 3-knots.

Example 7.3. We can construct simple 3 -knots which are $C$-algebraically fibered, but are not fibered, as follows. Let $K$ be a simple fibered 3 -knot. It is easy to see that there are a lot of simple algebraically non-fibered spherical 3-knots $K^{\prime}$ which are null cobordant. For example, consider the boundary of $S^{2} \times S^{2}-\operatorname{Int} D^{4}$ embedded in $S^{5}$ so that its Seifert form is isomorphic to

$$
\left(\begin{array}{cc}
0 & k \\
1-k & 0
\end{array}\right), \quad k \geq 1 \text {. }
$$

Then the simple 3 -knot $K \sharp K^{\prime}$ is $C$-algebraically fibered, since its Seifert form is algebraically concordant to that of $K$. However, $K \sharp K^{\prime}$ is not (algebraically) fibered.

Example 7.4. We can construct concordant, but not isotopic, non-spherical 3knots as follows. Let $K_{S}$ be a null cobordant stabilizer as in $\S 5$. Note that $K_{S}$ is a non-trivial 3-knot which is concordant to the trivial 3-knot. Then consider any simple fibered 3 -knot $K$ which is not spherical. Then the two simple fibered 3-knots $K \sharp K_{S}$ and $K$ are not isotopic, since the ranks of the second homology groups of their fibers are distinct. However, these knots are concordant. 
Example 7.5. For a simple 3-knot, the algebraic concordance class of a Seifert form depends on the choice of the Seifert manifold in general. For example, let $K$ be the trivially embedded $S^{1} \times S^{2}$ in $S^{5}$; i.e., $S^{4}$ is trivially embedded in $S^{5}$ and $S^{4}$ decomposes into $F_{0}=D^{2} \times S^{2}$ and $F_{1}=S^{1} \times D^{3}$ along $K$. Note that $F_{0}$ is a 1-connected Seifert manifold of $K$, while $F_{1}$ is a Seifert manifold which is not 1-connected. Then the Seifert forms with respect to $F_{0}$ and $F_{1}$ are not algebraically concordant, since the ranks of $H_{2}\left(F_{i}\right), i=0,1$, do not have the same parity.

\section{REFERENCES}

1. V. Blanlœil and F. Michel, A theory of cobordism for non-spherical links, Comment. Math. Helv. 72 (1997), 30-51. MR 98h:57049

2. S. Boyer, Simply-connected 4-manifolds with a given boundary, Trans. Amer. Math. Soc. 298 (1986), 331-357. MR 88b:57023

3. A. Durfee, Fibered knots and algebraic singularities, Topology 13 (1974), 47-59. MR 49:1523

4. J. A. Hillman, Simple locally flat 3-knots, Bull. London Math. Soc. 16 (1984), 599-602. MR 86a:57019

5. S. Kaplan, Constructing framed 4-manifolds with given almost framed boundaries, Trans. Amer. Math. Soc. 254 (1979), 237-263. MR 82h:57015

6. L. Kauffman, Branched coverings, open books and knot periodicity, Topology 13 (1974), 143160. MR 51:11532

7. C. Kearton, Some non-fibred 3-knots, Bull. London Math. Soc. 15 (1983), 365-367. MR 84m:57014

8. M. Kervaire, Les nœuds de dimensions supérieures, Bull. Soc. Math. France 93 (1965), 225271. MR 32:6479

9. M. Kervaire, Knot cobordism in codimension two, Manifolds-Amsterdam 1970 (Proc. Nuffic Summer School), Lecture Notes in Math. 197 (1971), Springer, Berlin, pp. 83-105. MR 44:1016

10. T. Lawson, Trivializing 5-dimensional h-cobordisms by stabilization, Manuscripta Math. 29 (1979), 305-321. MR 80i:57024

11. J. Levine, Knot cobordism groups in codimension two, Comment. Math. Helv. 44 (1969), 229-244. MR 39:7618

12. J. Milnor and D. Husemoller, Symmetric bilinear forms, Ergebnisse Math., Band 73, Springer, Berlin, Heidelberg, New York, 1973. MR 58:22129

13. U. Pinkall, Regular homotopy classes of immersed surfaces, Topology 24 (1985), 421-434. MR 87e:57028

14. F. Quinn, The stable topology of 4-manifolds, Topology Appl. 15 (1983), 71-77. MR 84b:37023

15. O. Saeki, On simple fibered 3-knots in $S^{5}$ and the existence of decomposable algebraic 3-knots, Comment. Math. Helv. 62 (1987), 587-601. MR 88k:57030

16. O. Saeki, Knotted homology 3-spheres in $S^{5}$, J. Math. Soc. Japan 40 (1988), 65-75. MR 89g:57032

17. O. Saeki, Cobordism classification of knotted homology 3-spheres in $S^{5}$, Osaka J. Math. 25 (1988), 213-222. MR 89g:57033

18. O. Saeki, Theory of fibered 3-knots in $S^{5}$ and its applications, J. Math. Sci. Univ. Tokyo 6 (1999), 691-756. MR 2001b:57058

19. O. Saeki, On punctured 3-manifolds in 5-sphere, Hiroshima Math. J. 29 (1999), 255-272. MR 2000h:57045

20. O. Saeki, A. Szücz, and M. Takase, Regular homotopy classes of immersions of 3-manifolds into 5-space, preprint, 2000.

21. C. T. C. Wall, Diffeomorphisms of 4-manifolds, J. London Math. Soc. 39 (1964), 131-140. MR 29:626 
Département de Mathématiques, Université Louis Pasteur Strasbourg I, 7 rue René Descartes, 67084 Strasbourg cedex, France

E-mail address: blanloeil@math.u-strasbg.fr

Faculty of Mathematics, Kyushu University, Hakozaki, Fukuova 812-8581, Japan

E-mail address: saeki@math.kyushu-u.ac.jp 